\title{
Experiences of breast cancer patients and helpful aspects of supportive-expressive group therapy: A qualitative study
}

Tânia Brandão ${ }^{1}$, Rita Tavares ${ }^{1}$, Marc S. Schulz $^{2}$ \& Paula Mena Matos ${ }^{1}$

${ }^{1}$ Faculty of Psychology and Education, University of Porto; Center for Psychology at University of Porto; Porto, Portugal.

2 Department of Psychology, Bryn Mawr College, Pennsylvania, USA.

*Corresponding author contact information: Dr. Paula Mena Matos, Rua Alfredo Allen 4200-135 Porto Portugal; Email: pmmatos@fpce.up.pt

Funding for this study was provided by a $\mathrm{PhD}$ Scholarship from the Portuguese Foundation for Science and Technology (FCT) granted to the first author (SFRH/BD/84436/2012).

Please Note: This is the author's version of the manuscript accepted for publication in European Journal of Cancer Care. Changes resulting from the publishing process, namely editorial editing, corrections, final formatting for publication, and other modifications resulting from quality control mechanisms may have been subsequently added.

A definitive version was subsequently published as: Brandão, T., Tavares, R., Schulz, M., \& Matos, P. M. (2019). Experiences of breast cancer patients and helpful aspects of supportive-expressive group therapy: A qualitative study. European Journal of Cancer Care, 27, e12830. Doi: $10.1111 /$ ecc. 13078 


\begin{abstract}
Objective: This study explored the experiences and perceived changes of breast cancer (BC) patients after participating in 16-weekly sessions of Supportive-Expressive Group Therapy (SEGT).

Methods: A semi-structured interview adapted from Elliott's Client Change Interview was carried out with 12 women (aged 33-60 years) with BC, about 6 months after completing the treatment.

Results: Content analysis identified four main themes: expectations and motivations to participate in SEGT, group processes and experiences, perceived changes enhanced by SEGT, and perceptions about the therapeutic relationship. The most helpful aspects of SEGT mentioned by participants were: the expression/normalization of feelings, thoughts, and reactions; the improvement of social support; and the learning opportunities obtained through sharing of experiences among participants. Additionally, participants mentioned that SEGT contributed to improve personal and social skills, such as the capacity to express emotions and the ability to establish satisfactory interpersonal relationships.
\end{abstract}

Conclusions: Based on the participants' experiences, SEGT seems to be an effective intervention to support women facing BC during the initial phase of cancer. The use of SEGT by health care professionals is encouraged, but the specific needs/problems of each group member should be carefully attended.

Keywords: supportive-expressive group therapy; breast cancer; patients' experiences; perceived benefits; 


\section{Introduction}

The experience of Breast Cancer (BC) exposes women to physical and psychosocial challenges (Campbell-Enns \& Woodgate, 2016; Koh, 2013). Researchers have focused their attention on testing the efficacy of psychosocial interventions offered to women with BC. Group therapies have been found to be effective in improving a wide range of psychosocial outcomes, including quality of life (QoL), anxiety and depression, social support perceptions, and coping strategies (Brandão \& Matos, 2015; Mustafa et al., 2013). The efficacy, however, has been found to vary with some studies showing no significant improvements (Brandão \& Matos, 2015). This can be explained by different types of interventions, diverse stages of the disease and different markers of adjustment. The mechanisms underlying efficacious interventions are largely unexplored (Moyer et al., 2012; Stanton et al., 2013). The lack of attention to process research in this area of intervention and the differences in efficacy found in existing interventions points to the need to explore how and for whom a specific intervention can be more effective. Important basic questions remain largely unanswered in the realm of psychosocial interventions for BC. How do patients perceive the therapy process and the components most linked to positive outcomes (Boulton et al., 2001; McLeod, 2001); what role does therapeutic alliance play (OmylinskaThurston \& Cooper, 2014); in what ways do patients' characteristics affect their response to therapy?

One common group therapy for women with BC is the Supportive-Expressive Group Therapy (SEGT), a semi-structured intervention aiming to improve social support, coping strategies, and healthy emotional processing. Efficacy studies have found benefits across a wide range of dimensions (e.g., QOL, psychological distress) (Butler et al., 2009; Classen et al., 2001; Giese-Davis et al., 2011; Reuter et al., 2010). Studies to date, however, have not captured patients' rich experiences in these interventions or how these experiences might be connected to outcomes 
(Giese-Davis et al., 2016). This is precisely the aim of this study. The focus of this study is on the perceived changes stimulated by this group therapy and on the potential processes underlying positive changes.

\section{Methods}

\section{Participants}

Participants were selected from an ongoing quasi-experimental study comparing SEGT (16 sessions) versus non-intervention (control group) for women with non-metastatic BC (ClinicalTrials.gov identifier: NCT02934815). Between March-2015 and February-2016, 26 women with BC agreed to participate. Three of them did not attend any sessions and an additional nine women dropped out (five of them after the $1^{\text {st }}$ session). The reasons given for dropping out included treatments side effects $(n=3)$, a sense that their needs may not be met in group therapy $(n=3)$, and having to cope with an illness in a family member $(n=1)$. One participant died of her disease; two provided no reason to drop out. A total of 13 women completed the group therapy and were invited to take part in this study. One patient declined to participate in the interview for this study, citing a lack of interest.

Participants $(N=12)$ had a mean age of 44.45 years $(S D=9.15$; range $33-60)$. On average, they completed high school $(\mathrm{M}=13$ years of education, $S D=3.13)$. The majority were married $(n=10)$ with a mean relationship length of 18 years $(S D=10.26)$. During SEGT, half of the women were on sick leave $(n=6)$, three were unemployed, two were working, and one was retired. All women had non-metastatic BC at entry and were diagnosed between two and 24 months $(M=10.92$ months; $S D=7.68$ ) prior to study entry. In terms of treatments, most completed chemotherapy $(n=11)$ and radiation therapy $(n=8)$, and most were undergoing or had completed hormonal therapy $(n=8)$. In terms of surgery, the majority had a mastectomy $(n=6)$, four had a lumpectomy, and one 
had a quadrantectomy. Half of the women also had breast reconstruction surgery. Participants attended, on average, twelve sessions of SEGT ( $M=11.67 ; S D=3.17$; range 6-15).

\section{Intervention}

SEGT is a semi-structured intervention that was originally developed by Spiegel, based on Yalom's existential psychotherapy, and emphasizes the need to recognize and work on existential concerns related to death, isolation, and meaning for women with metastatic BC (Spiegel, Bloom, \& Yalom 1981). Later, a briefer 16-session, manualized version of SEGT was developed for women with non-metastatic BC (Classen et al., 1993). In addition to focusing on existential concerns, SEGT addresses emotional, interpersonal, and practical challenges faced by cancer patients (Gore-Felton \& Spiegel, 2015). The objective of SEGT is to use the framework outlined above to discuss and work on relevant topics that arise naturally in each session and reflect the needs of the patients in each particular group (Classen \& Spiegel, 2011). The primary goals of SEGT are: facilitate mutual support among group members; promote openness and emotional expressiveness both within and outside the group; promote the integration of a changed self- and body-image into the patient's current view of the self; improve coping skills; improve social and family support; improve doctor-patient relationships; detoxify feelings around death and dying; promote reordering of life priorities; and enhance current quality of life. The main principles guiding treatment decision in sessions are to maintain the focus on cancer, promote the expression and exploration of feelings and thoughts, facilitate supportive interaction among group members, focus on personal and concrete issues, and facilitate active coping (Classen et al. 1993).

SEGT took place at an association to support women with breast cancer. Women participating in SEGT met weekly for 16 sessions for 90 minutes with a female therapist (the first author and sole facilitator of the SEGT) who was a clinical psychologist with previous experience 
in conducting group therapy for women with $\mathrm{BC}$, registered in the Order of Portuguese Psychologists, and a PhD student. To promote treatment fidelity, the therapist was carefully trained in SEGT, was supervised by a senior psychotherapist, and consulted with Dr. Spiegel to discuss therapy process and challenges during SEGT delivery.

\section{Data collection}

A semi-structured interview adapted from Elliott's Client Change Interview (Elliott \& Rodgers, 2008; Portuguese version: Sales et al., 2007) was used to obtain information about perceptions and experiences of the SEGT participants. This interview aims to explore patients' views of perceived changes after therapy, factors contributing to those changes, and helpful and unhelpful aspects of the therapy. The adapted version of the interview included questions focusing on patients' perspectives on the structure and length of therapy, potential benefits or drawbacks of SEGT, areas for improvement, and elements of therapeutic process including the group dynamic and the therapeutic relationship with the leader. The specific questions $(\mathrm{N}=31)$ asked during the interview are available in Supplemental data (Table 1). The semi-structured nature of the interview was designed so the interviewer could ask follow-up questions to clarify understanding or elicit elaboration. The interviewer could also alter the order of questions to improve the flow of the interview.

\section{Procedure}

The study was approved by the Ethical Committee of the Faculty of Psychology and Education Sciences at University of Porto. Participants were informed about the objectives of the study and had the opportunity to raise questions or concerns. Participants provided written informed consent to participate and to be audiotaped. Participation was voluntarily. No monetary 
compensation or any kind of incentive was offered. Interviews occurred in the facilities of an association to support women with breast cancer located in Porto. Each participant took part in a single interview in a private room. Interviews were conducted by a female psychologist and researcher (RT, the second author) with experience in conducting interviews with women with BC who was not directly involved in the therapy intervention. Interviews took place 5-6 months after completing the treatment. This time period was chosen to allow women time to cope with possible separation or loss experiences associated with the group ending and gain some perspective on their experiences. Interviews lasted, on average, 61 minutes $(S D=22.47$; range $=34-108 \mathrm{~min})$. Interviews were audiotaped and transcribed verbatim with the exception that identifying information (e.g., names) was removed.

\section{Data analysis}

Data were coded and analyzed using QSR NVivo 11 software according to guidelines for content analysis (Bardin, 1977). Categorization was performed in the following steps (Bardin, 1977): pre-analysis (interviews were transcribed verbatim and carefully read); encoding (themes were defined as the unit of analysis and enumeration was based on the presence or absence of a specific theme), categorization (themes were categorized, organized, and classified using both an inductive and deductive process), and, finally, interpretation (themes/codes were interpreted using an inferential process). The data extraction and the content analysis were conducted primarily and independently by the first and the second authors (the therapist and the interviewer). The last author (not involved in the therapy nor the interviews) read the transcribed interviews, discussed and reviewed with the other researchers the categorization themes process and the final coded themes. Final codes and themes were arrived at by consensus. 


\section{Results}

Analysis of the interview transcripts yielded four major themes: (1) expectations and motivations to participate in SEGT; (2) group processes and experiences; (3) perceived changes enhanced by SEGT; and (4) perceptions about the therapeutic relationship. Each theme was organized into different categories and subcategories as detailed in the following sections. Illustrative quotations have been selected to depict themes. Emerging themes, categories, and subcategories are presented in Figure 1.

\section{[INSERT FIGURE 1 ABOUT HERE]}

\section{Expectations and motivations to participate in SEGT}

This theme was conceptualized in terms of four categories: learning about other women's experiences $(n=6)$, altruism $(n=3)$, looking for social support $(n=3)$, and the group context $(n=1)$. Each category was organized into different subcategories as described below (highlighted by italics).

Most participants took part in SEGT with the hope of learning about other women's experiences. They hoped to share experiences $(n=3)$ associated with their diagnosis and the side effects of treatments. Participants thought that the group would give them the opportunity to talk about common concerns $(n=3)$ and to increase feelings of universality and normality $(n=3)$. Some participants seemed to be motivated primarily by desires for social support and for being altruistic. Regarding the first category, participants expected that SEGT could increase their social support $(n=2)$ and promote their feeling of being part of the support center $(n=1)$. Regarding altruism, some participants expressed a desire to contribute to the research study $(n=2)$ and others 
found meaning in helping others $(n=2)$. One was primarily motivated to participate in the SEGT because of the group context $(n=1)$.

The fact that I knew that it was going to be a group where we all had something in common and that we would all speak the same language... (P5, 41 years). The fact that I knew that it was not going to be just me and the psychologist, right? If so, I would feel a little bit intimidated, so the fact that I would be together with more people [in the group] gave me a sense of comfort... (P5, 41 years).

\section{Group processes and experiences}

This theme was organized into two categories: helpful/positive aspects $(n=12)$ and unhelpful/problematic aspects $(n=10)$. Examples of all helpful/positive and unhelpful/problematic aspects and corresponding quotations are presented in Supplemental data (Table 2). The most commonly perceived positive aspects were related to the opportunity to learn about and share experiences with other women $(n=12)$, to improve their social support $(n=10)$, and to realize that they were not alone in their difficulties (subcategory: universality and feelings of normalization) $(n=10)$ :

Feeling that other women have gone through the same experience as us, we feel a lot less alone (...) And I think that in terms of normalizing it is much easier when we hear other women saying the same thing we are thinking. (P12, 42 years).

Additionally, participants mentioned other helpful/positive aspects of SEGT program such as a sense of empowerment $(n=8)$, catharsis and emotional expression $(n=7)$, altruism $(n=4)$, improvement of interpersonal relationships $(n=3)$, instillation of hope $(n=2)$ and openness $(n=2)$. 
With regards to unhelpful/problematic aspects, participants highlighted the challenges associated with sharing difficult issues, such as talking about issues related to couple intimacy $(n=9)$, the difficulty in dealing with different opinions and needs $(n=3)$, leaving children at home when they are in the group $(\mathrm{n}=1)$, and the discomfort of thinking about or dealing with (the possibility of) recurrence $(n=2)$ :

I found it difficult to talk during the whole group (...) because I was not ready,

I did not want to talk about it either, because maybe I did not know very well what I wanted to say about it. I had first to figure it out so I could talk about it(...) the worst part of being in a group for me was not being able to open up to the group about more intimate issues. (P6, 33 years)

Participants' perceptions about positive and problematic aspects of SEGT also touched on structural and functional aspects of the therapy, namely in terms of group size $(n=4)$, organization $(n=4)$, duration and frequency $(n=10)$, structure $(n=8)$, suggestions for improvement $(n=4)$, meeting and adapting to unfamiliar persons $(n=2)$, members dropping out of the group $(n=2)$, and absences and lack of group cohesion $(n=1)$. Regarding group size, two participants considered the number of members in the group to be appropriate, while two other women felt a larger group would be helpful. Four participants considered the organization of the group adequate; ten perceived the frequency and duration of sessions to be satisfactory, but seven participants would have preferred to have more and longer sessions. The main reasons participants gave for more time were: to have the chance to talk about and discuss more issues; to have time to finish some issues in the sessions; to use sessions more effectively after becoming comfortable talking and expressing thoughts and feelings; and the desire to continue to have support until completing medical treatments. 
Regarding the structure of the group, some participants viewed the unstructured format of SEGT favorably $(n=4)$, while some would have preferred a more structure and directive intervention $(n=4)$. In the suggestions component, some participants expressed a desire to have themes for each session to organize discussion. Other suggestions included more talking about faith and greater focus on issues related to intimacy.

\section{Perceived changes enhanced by SEGT}

Most participants $(n=9)$ perceived some changes in themselves after participating in SEGT. These comprised not only personal changes $(n=9)$ but also social changes $(n=6)$. Personal changes included a greater capacity to express emotions $(n=6)$ not only in the group but also within other interpersonal relationships (e.g., children, husbands). The participants also described being more comfortable expressing emotions stimulated by a wide variety of everyday contexts, not just those related to $\mathrm{BC}$ experience. Participants felt that the group experience fostered a greater recognition of and ease with their own emotions and thoughts:

I think that now I can talk more [about the cancer] ... I am not so afraid. At first, I did not want to talk about it, but I think that after [the group] I started to talk more about it... (P7, 39 years)

I think I have been integrating a series of negative emotions, this is a long learning process and it is not yet finished, but I think that it was important for me to talk about (P12, 42 years).

Some women reported changes related to reordering of life priorities $(n=3)$, developing a more positive view of life $(n=2)$, and becoming more optimistic $(n=1)$ :

[We] put our disease in perspective... and that made us re-think everything else... (P12, 42 years) 
Some participants reported improvements in coping with practical issues $(n=2)$, self-care skills $(n=1)$, and self-esteem or a sense of security $(n=2)$. In terms of social changes, some participants reported improvements in interpersonal relationships $(n=5)$ and social support $(n=1)$ :

Ifelt that the group has helped me to face this situation with more humility and more serenity. That was the main advantages (...) I improved my ability to relate to others ( $\mathrm{P} 4,45$ years).

Three participants did not perceive any changes following SEGT: This disease never affected me much... I had a good team that treated my cancer and I had the support of every people... (P8, 55 years).

\section{Therapeutic relationship}

Three main categories were found: characteristics of the therapist $(n=9)$, leadership $(n=8)$, and promotion of personal skills $(n=11)$.

The most important characteristics of the therapist reported by the participants were empathy $(n=4)$ and active listening $(n=3)$. Some participants also highlighted the importance of the therapist's capacity to establish closeness $(n=2)$ and to promote a sense of security $(n=1)$ or serenity $(n=1)$ :

I think it was [important] her empathy and trustworthiness... We felt that the person was there listening to us, right? (P6, 33 years).

The fact that she has created this empathy with me and with others, I speak for myself, the fact that she has created such a great empathy, gave me freedom to talk about what I did not want to talk about... (P3, 44 years). 
Participants' perceptions regarding the issue of leadership varied. While some participants expressed a desire for more leadership $(n=4)$, other participants believed that the therapist had demonstrated adequate leadership $(n=4)$, by allowing them to talk freely about anything that was important for them in that moment:

Sometimes it was necessary to... be almost like a judge and say, "Your time is over, now let's hear ..." I did not say that she did not do it, because she did, but there were times when it was already too much the same person talking about something. I know that she was respecting the space of each woman... (P6, 33 years).

She gave us the freedom to talk about everything we wanted. Therefore, she never imposed a theme on us. And the fact of giving us this freedom [was important] ... (P3, 44 years).

Finally, participants recognized that the therapist contributed to helping them refine their personal skills $(n=11)$, specifically in terms of emotional expression $(n=9)$ and coping strategies $(n=2)$ :

(...) She gave us the clues we needed, to act with security, I think she was very confident about what she was saying, so we could do the things on our own... in the context of our family, in the context of our friends, in our environment (P4, 45 years).

Yes, I think so [she helped me to express what I was feeling] (...) In fact, I remember her encouraging me several times, to talk, right? Because she realized, right? "I would like to hear P9....". Because she realized that I did not ... She realized, I also told her that I did not like to talk, that's it. And she was pushing a little, I noticed that... (P9, 37 years). 


\section{Discussion}

This study aimed to gain an in-depth understanding of patients' perceptions about their experiences in 16 weeks of SEGT for women with BC. The goals were to give voice to participant's views of the nature and processes of change during SEGT as well as to highlight patients' perceptions of SEGT structure and functioning. Overall, the analyses indicate that, from the perspective of participants, SEGT is an effective intervention for women facing BC.

Participants' expectations about and motivations for taking part in SEGT seemed to be fulfilled. Most women hoped to learn along with other women about experiences with BC and to gain more social support. After SEGT, women repeatedly identified sharing experiences, feelings of universality and normalization, and social support as the most helpful and positive therapeutic aspects of SEGT. Participants also identified other beneficial components of their SEGT participation: the sense of empowerment they developed as a result of sharing information with and learning from peers, the value of being able to express their emotions openly with group members, the benefits to themselves of helping others in the group, the opportunities to practice relational skills that could be generalized outside of the therapy, and the instillation of hope. These processes are in accordance with key group therapeutic factors described by Yalom and Leszcz (Yalom \& Leszcz, 2005) and were identified as important aspects in other studies with cancer patients (Bell et al., 2010; Reuter et al., 2010). These findings provide support for the rationale underlying the potential utility of SEGT with women with non-metastatic breast cancer. Sharing feelings and experiences are considered important aspects of self-disclosure, which is viewed as a key driver of change in group therapy and a critical mechanism for promoting connections and strength of a group relationship (Bloch \& Crouch, 1985; Dierick \& Lietaer, 2008). 
The group context created by SEGT seemed to act as a secure and safe environment that facilitated a range of learning opportunities for participants: talking about themselves and their response to cancer, sharing their experiences, expressing their emotions and thoughts freely, and feeling reassured by understanding that their emotions, thoughts, and reactions were 'normal' and that they were not alone. These core therapeutic processes and proximal results are similar to those that have been identified in quantitative studies of SEGT efficacy (Cleary \& Stanton, 2015; Manne et al., 2008). Moreover, given the most helpful aspects identified (i.e., expression and normalization of feelings, thoughts, and reactions as well as all the learning opportunities and information), these results seem to suggest that SEGT can be particularly useful during the initial phase of cancer trajectory in which patients are confronted with their lack of knowledge, fears of treatments, doubts about managing treatment side effects, and worries about mortality (Campbell-Enns \& Woodgate, 2016).

The acquisition of key skills and positive changes linked to SEGT were identified by most participants. The most emphasized changes included a greater capacity to express emotions, improvements in their broader social support network (outside of the group) and reordering of life priorities. Each of these changes are consistent with the main objectives of SEGT (Brandão \& Matos, 2015; Classen et al., 1993) and are similar to the outcomes reported in other studies (e.g., Ahlberg \& Nordner 2006). It is now widely recognized that the way women with BC regulate their emotions influences their psychological adjustment to the disease (Brandão et al., 2016). Participants in this study recognized that, after SEGT, they became more capable of expressing their emotions to others. This increased ease of emotional expression, particularly in regard to challenging emotions, may be tied in important ways to the perceptions of improved social support for women in SEGT. More comfort expressing emotions and thoughts is believed to contribute to decreasing a sense of isolation from others. Moreover, participants may learn how to "detoxify" 
certain emotions and topics of conversation in the group and be able to generalize it to their social contexts. Maintaining conversations with close others without being emotionally overwhelmed may facilitate connections with others and increase the likelihood that key needs will be met. It will be important in future research to explore whether SEGT is particularly useful for women struggling with less adequate emotion regulation strategies or lacking social support.

It is notable that three participants (out of 12) did not identify any specific changes that they thought were associated with SEGT. One of them only attended eight sessions (half of the program sessions) which might have limited the benefits obtained. The two remaining attended almost all of the sessions but they highlighted how they seemed to cope well with the disease from the beginning, emphasizing their altruistic reasons to participate in the SEGT. However, like most therapies, it is likely that SEGT does not work equally well or in the same way for all patients. The results of this study suggest that process-oriented research intended to understand the healthpromoting benefits of SEGT should also attend to critical moderators that may determine why some benefit more than others and what types of patients are most likely to be aided by particular mechanisms. In addition, the study of efficacy of intervention programs could benefit from including the perception of close others regarding changes of the targeting participants. In some cases, not reporting changes could be associated to self-protecting mechanisms related to the need of maintaining a sense of competent self, but change could be identified by other figures.

The patients' comments also point to the critical role of the therapeutic relationship with the group leader. Analyses indicated that most participants trusted the therapist and expressed appreciation for the therapist's professional and personal characteristics. These characteristics are valued by clients (Johnson \& Caldwell, 2011) and recognized as important for therapy outcomes across a wide range of treatments (Norcross \& Wampold, 2011). Moreover, they are essential to promote participants' disclosure of distressing emotions and thoughts (Angus \& Kagan, 2007), a 
central goal of SEGT. Thus, the safe and non-judgmental atmosphere advocated by SEGT seems to be essential to making it beneficial to participants. The results suggest how important it is to train therapists with skills that build trust and help facilitate exploration and sharing by patients. Some participants raised concerns about the non-directive role of the therapist. These participants stated that they were expecting a more active role from the therapist in terms of talking more, managing the group, and giving more advice. However, other participants viewed the nondirectiveness of the therapist as a positive aspect of the group since the participants themselves could decide what was important to focus on and freely talk about their preferred topics. These comments are particularly notable since SEGT is intentionally designed to allow the group to decide what the focus of each group should be (Classen et al., 1993). The therapist is viewed as a facilitator of group processes, but the hope is that advice, information and coping strategies will be offered mostly from other group members since they have first-hand experience in meeting similar challenges.

Some of the participants who would have preferred a more directive approach were those who had the most difficulties opening up and were those who reported the fewest gains from SEGT. Their desire to passively receive practical advice and information may reflect some of their difficulties with directly confronting their distress and challenges. These results seem to suggest that women with these specific characteristics might benefit less from a group therapy format since it seems to create an additional strain. On the other hand, participating in an intervention that targets individual's difficulties with processing challenging experiences and expressing discomfort could give them critical opportunities to work on and improve those skills. It is also possible that exposure to other women who are more comfortable and agile in expressing themselves may provide a helpful model for these individuals. What differentiates SEGT from other types of support groups (i.e., unstructured format, non-directive approach) may lead to variations in responses among 
participants. SEGT therapists need to be attentive to these variations in response. It might be useful in future applications of SEGT to proactively monitor these "cautious" women and to consider ways of adapting the approach to meet these women more effectively where they are at in their process of adapting to their experience.

Finally, some suggestions from the participants emerged regarding group structure and functioning. The suggestions were not consistent across participants; some are in fact contradictory. For these reasons, it is particularly important to exercise caution in drawing any definite conclusions regarding the ideal number of sessions, length of intervention, or structure of the intervention. Some participants found that group size was adequate while others thought that having a larger group would be beneficial. In fact, the ideal number of participants as described in the treatment manual for Brief SEGT is between eight and ten women (Classen et al., 1993). This group size was not attained because some participants dropped out after first session and others missed some sessions because of cancer treatments. By having a reduced number of women per group, the number of shared experiences and the varied coping strategies described are reduced, which may limit the benefits derived from SEGT. However, in a small group, each woman has the chance to participate and share more. Results from this study suggest that SEGT has benefits regardless of the number of participants.

Results from this study should be interpreted with caution given some of its limitations. As is typical of qualitative studies, we analyzed responses from a small number of participants $(N=$ 12). It is important to note that the first author was also the main therapist for all the SEGT groups (but was not present in the interviews), which may have biased the understanding and framing of interview responses in some ways. To reduce potential bias as well as eliminate pressure for participants to please the therapist, interviews were conducted by another independent researcher (not involved in the therapy sessions). Also, analysis of responses was performed by multiple 
researchers including one who did not have any contact with patients. The findings presented here are dependent on participants' recall and their capacity for insight and reflection. It is possible that participants attributed some changes to group participation that were actually influenced by other factors such as their response to treatment. A wide range of positive and more problematic aspects of experiences with SEGT were identified. However, it is important to note that the women who participated in the study volunteered to take part in this research, which might have influenced the results presented here. The study did not interview participants who dropped out of groups, which might have provided additional useful information.

In sum, SEGT seems to be an intervention useful for patients with non-metastatic breast cancer. Thus, health care professionals are encouraged to use SEGT within this context. However, they should be aware of the need to explore expectations and motivations of participants in order to assess the appropriateness of SEGT for a specific patient. Moreover, health care professionals should implement SEGT by taking into consideration the specific needs and difficulties of each patient. They should not only facilitate interactions among member groups but also closely monitor those women who present more difficulties in the process of adaptation to breast cancer.

\section{Conflict of interest}

The authors declare that there is no conflict of interest.

\section{References}

Ahlberg, K., \& Nordner, A. (2006). The importance of participation in support groups for women with ovarian cancer. Oncology Nursing Forum, 33, 53-61. https://doi.org/10.1188/06.ONF.E53-E61

Angus, L., \& Kagan, F. (2007). Empathic relational bonds and personal agency in psychotherapy: 
Implications for psychotherapy supervision, practice, and research. Psychotherapy: Theory, Research, Practice, Training, 44, 371-377. https://doi.org/10.1037/0033-3204.44.4.371

Bardin, L. (1977). Análise de Conteúdo [Content Analysis] (4th ed.). Lisboa: Edições 70.

Bell, K., Lee, J., Foran, S., Kwong, S., \& Christopherson, J. (2010). Is there an “ideal cancer" support group? Key findings from a qualitative study of three groups. Journal of Psychosocial Oncology, 28, 432-449. https://doi.org/10.1080/07347332.2010.488140

Bloch, S., \& Crouch, E. (1985). Therapeutic factors in group psychotherapy. Oxford: Oxford University Press

Boulton, M., Boudioni, M., Mossman, J., Moynihan, C., Leydon, G., \& Ramirez, A. (2001). ?Dividing the desolation?: Clients views on the benefits of a cancer counselling service. Psycho-Oncology, 10, 124-136. https://doi.org/10.1002/pon.494

Brandão, T., \& Matos, P. M. (2015). Eficácia das intervenções psicológicas em grupo dirigidas a mulheres com cancro da mama: uma revisão sistemática. Revista Portuguesa de Saúde Pública, 33, 98-106. https://doi.org/10.1016/j.rpsp.2015.01.001

Brandão, T., \& Matos, P. M. (2015). Terapia breve de apoio e expressividade emocional para mulheres com cancro da mama: Manual de intervenção em grupo. Porto: FPCEUP.

Brandão, T., Tavares, R., Schulz, M. S., \& Matos, P. M. (2016). Measuring emotion regulation and emotional expression in breast cancer patients: A systematic review. Clinical Psychology Review, 43, 114-127. https://doi.org/http://dx.doi.org/10.1016/j.cpr.2015.10.002

Butler, L. D., Koopman, C., Neri, E., Giese-Davis, J., Palesh, O., Thorne-Yocam, K. a, ... Spiegel, D. (2009). Effects of supportive-expressive group therapy on pain in women with metastatic breast cancer. Health Psychology, American Psychological Association, 28, 579587. https://doi.org/10.1037/a0016124

Campbell-Enns, H. J., \& Woodgate, R. L. (2016). The psychosocial experiences of women with 
breast cancer across the lifespan: A systematic review. Psycho-Oncology, 13, 112-121. https://doi.org/10.1002/pon.4281

Classen, C., Butler, L. D., Koopman, C., Miller, E., DiMiceli, S., Giese-Davis, J., ... Spiegel, D. (2001). Supportive-expressive group therapy and distress in patients with metastatic breast cancer. Archives of General Psychiatry, 58(5), 494-501 https://doi.org/10.1001/archpsyc.58.5.494

Classen, C., Diamond, S., Soleman, A., Fobair, P., Spira, J., \& Spiegel, D. (1993). Brief supportive-expressive group therapy for women with primary breast cancer. A treatment manual. Stanford: Stanford School of Medicine.

Cleary, E. H., \& Stanton, A. L. (2015). Mediators of an Internet-based psychosocial intervention for women with breast cancer. Health Psychology, 34, 477-485. https://doi.org/10.1037/hea0000170

Dierick, P., \& Lietaer, G. (2008). Client perception of therapeutic factors in group psychotherapy and growth groups: An empirically-based hierarchical model international journal of group psychotherapy. International Journal of Group Psychotherapy, 58, 203-230. doi: 10.1521/ijgp.2008.58.2.203.

Elliott, R., \& Rodgers, B. (2008). Client change interview schedule. Unpublished research instrument, University of Strathclyde, Glasgow.

Elliott, R. (2012). Qualtative methods for studying psychotherapy change processes. In D. Harper \& A. R. Thompson (Eds.), Qualitative research methods in mental and health psychotherapy. A guide for students and practitioners. (pp. 69-82). Chichester, UK: Wiley John Wiley \& Sons, Inc.

Giese-Davis, J., Brandelli, Y., Kronenwetter, C., Golant, M., Cordova, M., Twirbutt, S., ... Spiegel, D. (2016). Illustrating the multi-faceted dimensions of group therapy and support 
for cancer patients. Healthcare, 4(3), 1-14. https://doi.org/10.3390/healthcare4030048

Giese-Davis, J., Collie, K., Rancourt, K. M. S., Neri, E., Kraemer, H. C., \& Spiegel, D. (2011).

Decrease in depression symptoms is associated with longer survival in patients with metastatic breast cancer: A secondary analysis. Journal of Clinical Oncology, 29, 413-420. https://doi.org/10.1200/JCO.2010.28.4455

Gore-Felton, C., \& Spiegel, D. (2015). Group psychotherapy for medically ill populations. In B. S. Fogel, \& D. B. Greenberg (Eds), Psychiatric care of the medical patient, (pp. 230-242). Oxford: Oxford University Press.

Johnson, L. A., \& Caldwell, B. E. (2011). Race, gender, and therapist confidence: effects on satisfaction with the therapeutic relationship in MFT. The American Journal of Family Therapy, 39, 307-324. https://doi.org/10.1080/01926187.2010.532012

Koh, K. B. (2013). Psychosocial aspects of breast cancer: Focus on interventions. In K. B. Koh (Ed.), Somatization and psychosomatic symptoms (pp. 239-257). New York, NY: Springer New York. https://doi.org/10.1007/978-1-4614-7119-6

Manne, S. L., Winkel, G., Rubin, S., Edelson, M., Rosenblum, N., Bergman, C., ... Rocereto, T. (2008). Mediators of a coping and communication-enhancing intervention and a supportive counseling intervention among women diagnosed with gynecological cancers. Journal of Consulting and Clinical Psychology, 76, 1034-1045. https://doi.org/10.1037/a0014071

McLeod, J. (2001). The contribution of qualtative resarch to evidence-based counselling and psychotherapy. In N. Rowland \& S. Goss (Eds.), Evidence-based counselling and psychological therapies. Research and applications (Vol. 179, pp. 111-126). London: Routledge. https://doi.org/10.1192/bjp.179.2.184-a

Moyer, A., Goldenberg, M., Hall, M. A., Knapp-Oliver, S. K., Sohl, S. J., Sarma, E. A., \& Schneider, S. (2012). Mediators of change in psychosocial interventions for cancer patients: 
a systematic review. Behavioral Medicine, 38, 90-114.

https://doi.org/10.1080/08964289.2012.695412

Mustafa, M., Carson-Stevens, A., Gillespie, D., \& Edwards, A. G. (2013). Psychological interventions for women with metastatic breast cancer. In A. G. Edwards (Ed.), Cochrane Database of Systematic Reviews. Chichester, UK: John Wiley \& Sons, Ltd. https://doi.org/10.1002/14651858.CD004253.pub4

Norcross, J. C., \& Wampold, B. E. (2011). Evidence-based therapy relationships: Research conclusions and clinical practices. Psychotherapy, 48, 98-102. https://doi.org/10.1037/a0022161

Omylinska-Thurston, J., \& Cooper, M. (2014). Helpful processes in psychological therapy for patients with primary cancers: a qualitative interview study. Counselling and Psychotherapy Research, 14(2), 84-92. https://doi.org/10.1080/14733145.2013.813952

Reuter, K., Scholl, I., Sillem, M., Hasenburg, A., \& Härter, M. (2010). Implementation and benefits of psychooncological group interventions in german breast centers: A pilot study on supportive-expressive group therapy for women with primary breast cancer. Breast Care, 5(2), 91-96. https://doi.org/10.1159/000297739

Sales, C., Gonçalves, S., Silva, I., Duarte, J. Sousa, D., Fernandes, E. (2007). Portuguese adaptation of qualitative change process instruments. Paper presented at the European Chapter Annual Meeting of the Society for Psychotherapy Research, Madeira, Portugal. Spiegel, D., Bloom, J. R., \& Yalom, I. (1981). Group Support for Patients With Metastatic Cancer. Archives of General Psychiatry, 38, 527-533. https://doi.org/10.1001/archpsyc.1980.01780300039004

Spiegel, D., \& Spira, J. (1991). Supportive-expressive group therapy: A treatment manual of psychosocial intervention for women with recurrent breast cancer. Stanford: Stanford 
School of Medicine.

Stanton, A. L., Luecken, L. J., MacKinnon, D. P., \& Thompson, E. H. (2013). Mechanisms in psychosocial interventions for adults living with cancer: opportunity for integration of theory, research, and practice. Journal of Consulting and Clinical Psychology, 81, 318-35. https://doi.org/10.1037/a0028833

Waters, E. A., Liu, Y., Schootman, M., \& Jeffe, D. B. (2013). Worry about cancer progression and low perceived social support: Implications for quality of life among early-stage breast cancer patients. Annals of Behavioral Medicine, 45, 57-68. https://doi.org/10.1007/s12160012-9406-1

Yalom, I. D., \& Leszcz, M. (2005). Theory and practice of group psychotherapy (5th ed.). New York: Basic books. 
Figure 1. Visual description of the emerging themes, categories, and subcategories. 